\title{
Altered Behaviour: The Performative Nature of Manufacture Chainsaw Choreographies + Bandsaw Manoeuvres
}

\author{
Emmanuel Vercruysse ${ }^{1}$, Zachary Mollica ${ }^{2}$ and Pradeep Devadass ${ }^{3}$ \\ ${ }^{1 / 2}$ Design + Make, Architectural Association School of Architecture. London, UK \\ ${ }^{3}$ Individualized Production, RWTH Aachen University. Aachen, DE
}

\begin{abstract}
This paper explores the ventures of the Robotic Fabrications AA Visiting School. Cultivating and implementing radical and innovative modes of architectural fabrication, we borrow from neighbouring creative fields such as choreography, performance and martial arts - efficient code being infected with an exciting spatial complexity and positing an artistic engagement within the world of physical production.
\end{abstract}

\section{Introduction}

The Robotic Fabrications Visiting School ${ }^{1}$ operates as an experimental branch of the Architectural Association's Design + Make programme - cultivating and implementing radical and innovative modes of architectural production. We foster a deep interest in the technical aspects of robotics while ultimately looking to engage it in a wider spatial and cultural discourse. Merging expert knowledge of timber construction with 6-axis robotic fabrication - the creative potential of prototyping complex timber structures has been explored through the progressive manufacture of a Foundry for Hooke Park.

Traditional tools and techniques for making are hijacked, re-invented and applied as innovative processes for architecture. Our two weapons of choice - a bandsaw and chainsaw - gain an augmented level of precision and control when wielded by the Kuka KR150. In converting these tools into end-effectors, their performance is altered. Through rigorous physical testing, we prototype intricate connection details, and apply the extraordinary precision and flexibility of multi-axis robotic machining while ensuring design strategies are firmly rooted within tradition.

If the behaviour of the hijacked tool has transformed, then so has our space of production. As traditional fabrication principles undergo a digital re-interpretation through the implications of robotics - they deviate from the tightly controlled motion paths of

${ }^{1}$ Robotic Fabrications is run by director Emmanuel Vercruysse and tutor Zachary Mollica with the robotics team of Pradeep Devadass, Gary Edwards and recently, Michael Arnett. Authors Emmanuel and Zachary are respectively a co-director and studio tutor for the AA's Design + Make programme. At the time of the work presented, author Pradeep Devadass was AA Hooke Park's Robotics Developer as part of a collaboration between the AA, UWE and KTP. 
industrial applications into the realm of staged events. Borrowing from neighbouring creative fields such as choreography, performance and martial arts, efficient code is infected with an exciting spatial complexity and posits an artistic engagement within the world of physical production. Although the fabrication of complex architectural components remains the core driver, the performative nature of manufacturing has become a key driver in our augmented space of production.

\subsection{The Foundry}

While timber sourced from the surrounding woodland has served as the primary working material for the architectural constructs developed in Hooke Park, recent investigations into metal casting instigated the development of a Foundry for the campus. The outcome of the first workshop, 'Chainsaw Choreographies,' is an opening move. Four overhanging Douglas fir beams interwoven and supported on three naturally formed tree forks set out a precise circular plan. The design evolves incrementally, through 'Bandsaw Manoeuvres,' 10 distinct components were fabricated which together form a complex ring beam which will receive the structure's eventual shell.

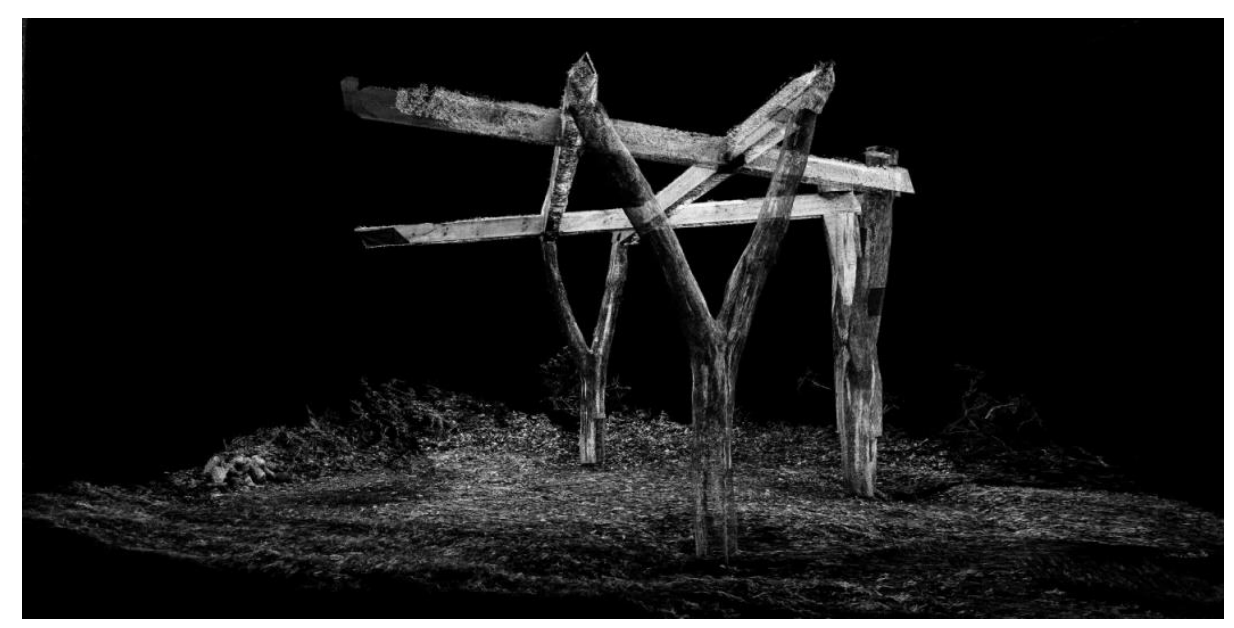

Fig. 1. Photogrammetric 3D scan of the Foundry after 'Chainsaw Choreographies'. This precise survey confirmed the accuracy of fabrication and served as a base for further development. 


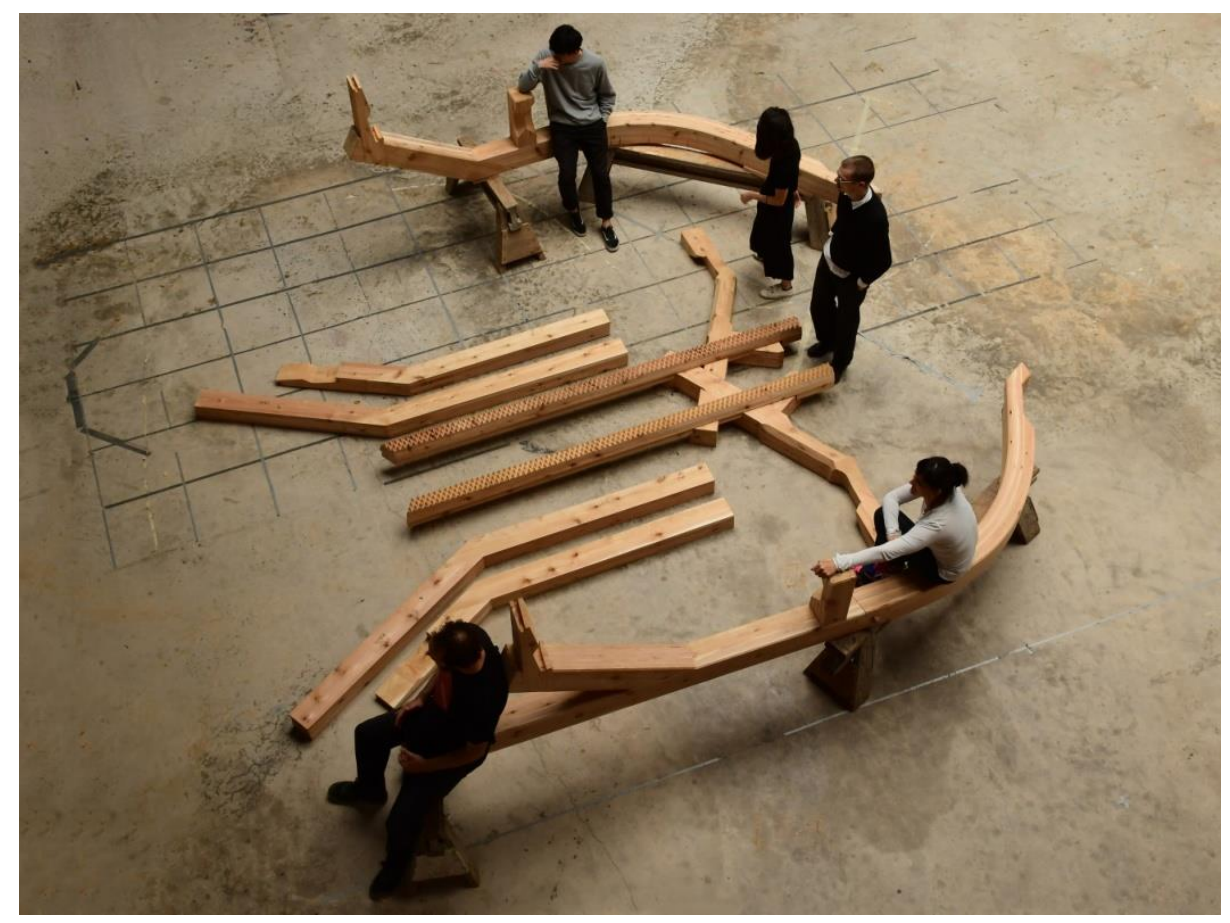

Fig. 2. 'Bandsaw Manoeuvres' participants inhabit the 10 components of the ring beam.

\subsection{Material Library}

Headquartered in Hooke Park, we are embedded within our primary material library the forest itself. Beyond its immediate proximity, timber is chosen as the main material for our experiments due to its inherently dynamic properties. The form of each standing tree is a distinct composition of grain that has grown in direct response to its environs. This form governs how each piece of timber derived from the tree reacts to subsequent operations. Through careful understanding of this variation, it can be accounted for within our methods. "It is important to remember wood is a living thing - one must understand its properties and not oppose them" [1].

In the development of the Foundry, timber has been used in various formats: large rectilinear beams; naturally formed ' $\mathrm{Y}$ ' shaped forks ${ }^{2}$; and bespoke stocks made through a variety of lamination techniques. The timber used throughout has been 'green' - with a moisture content between $20-70 \%$ and therefore an increased predisposition to movement. Restricted by the quantity of energy they could employ to join timbers, traditional craftsmen developed methods which engaged closely with the material's grain and embodied information. While mechanical fasteners have become standard practice for

\footnotetext{
${ }^{2}$ The authors, as faculty for the AA's Design + Make programme, gained significant expertise in working with complex inherent timber forms through the Wood Chip Barn project (2016). 'Chainsaw Choreographies' expanded on the extensive workflows established [2].
} 
jointing timber assemblies due to their ease and predictable performance, Robotic Fabrications explores the development of complex timber to timber connections inspired by traditional Japanese joinery.

\section{$2 \quad$ Altering the Behaviour of Tools}

\subsection{Act 1: The Chainsaw, Act 2: The Bandsaw}

In the laboratory environment of Robotic Fabrications, we foster a critical approach towards design-through-making where making is central to the act of design and learning central to the act of making. The core objective of these 10-day workshops is an exploration - to unravel, discover and assemble an in-depth knowledge of the newly installed end-effector. The pace is furious, producing 10 components in 10 days this year, and through the reinvention and hybridisation of tools and techniques, unique methods of fabrication are exposed.

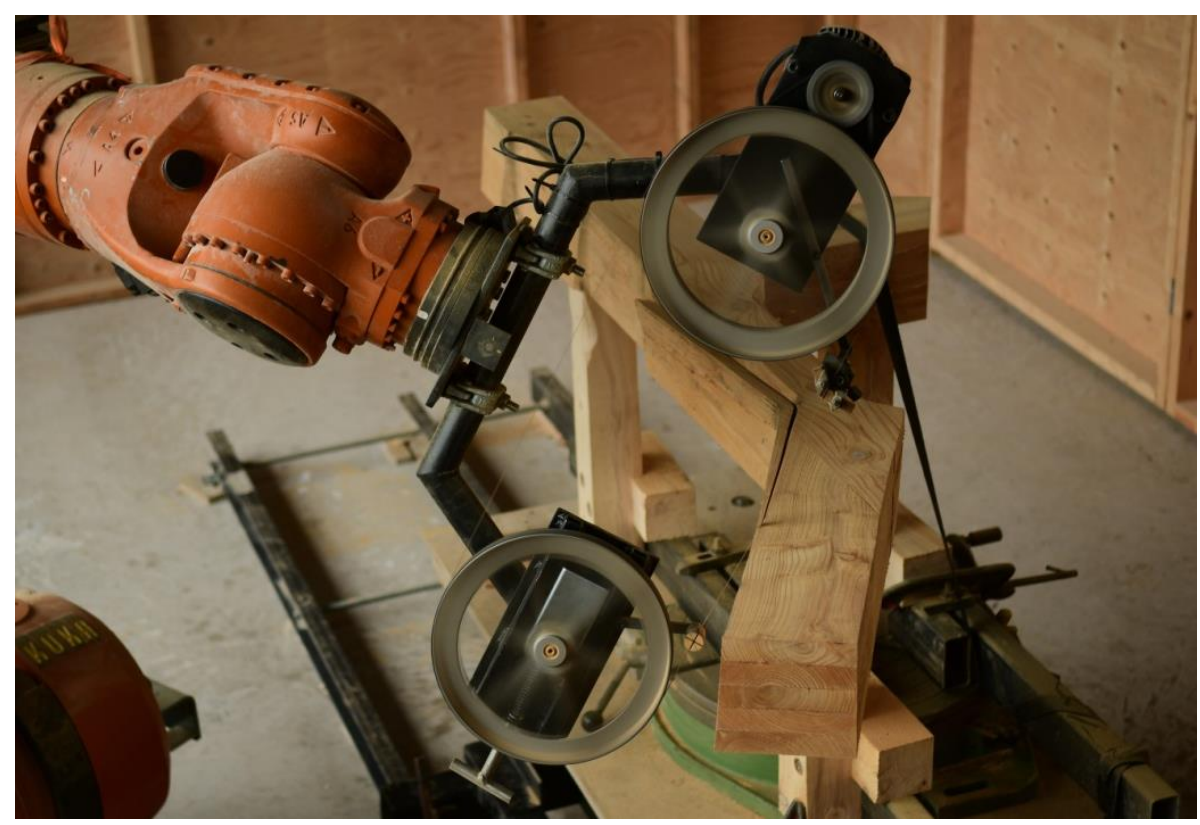

Fig. 3. Weapon of choice - bandsaw end effector used during 'Bandsaw Manoeuvres'

The selection of tool to be employed for each task is significant. Beyond a requirement to achieve a particular duty well, tools are chosen for their temperament, complications and limitations. Borrowed from the world we operate in daily - the wood workshop and surrounding forest - the bandsaw and the chainsaw have become our weapons of choice. Though each of these tools has been applied as a robotic end-effector in previous investigations, they have been employed typically as a means of achieving component shape - with less emphasis given to material properties and the rich history 
of craftspeople deriving timber joinery directly from these characteristics. In a work titled '7x Stool' [3/4], Pawlofsky and Weissmahr employed a chainsaw end-effector to cut a pair of stools and a small table from a single piece of round timber. With priority given to the gesture of this act, the anisotropic nature of timber remains neglected in the finished pieces. A bandsaw end-effector has been applied to timber fabrication within two recent projects: Philip Yuan and Hua Chai's 'Robotic Wood Tectonics' [5], and Ryan Luke Johns and Nicholas Foley's 'Bandsawn Bands' [6]. Each demonstrates the ability of this continuous blade to create curved geometries, and while neither engages significantly with timber to timber joinery, the latter's intent to derive forms from wood's own nonstandard grain configuration adds a richness to the work.

Robotic Fabrications use of these two tools is distinguished by two primary motives: to develop complex timber to timber connections rooted in tradition; and to explore performative manufacturing through analogy between robotic trajectories and human motion. Fundamentally setting apart the work presented is its location in Hooke Park. With a diverse team made up of craftspeople, designers, engineers, roboteers, a forester and more specialist knowledge is introduced from each. Working closely with Hooke Park's Workshop Manager Charlie Corry Wright and Forester Christopher Sadd to attach these tools to the robotic arm we begin to dissect and analyse the shifts in behaviour implied by their operator.

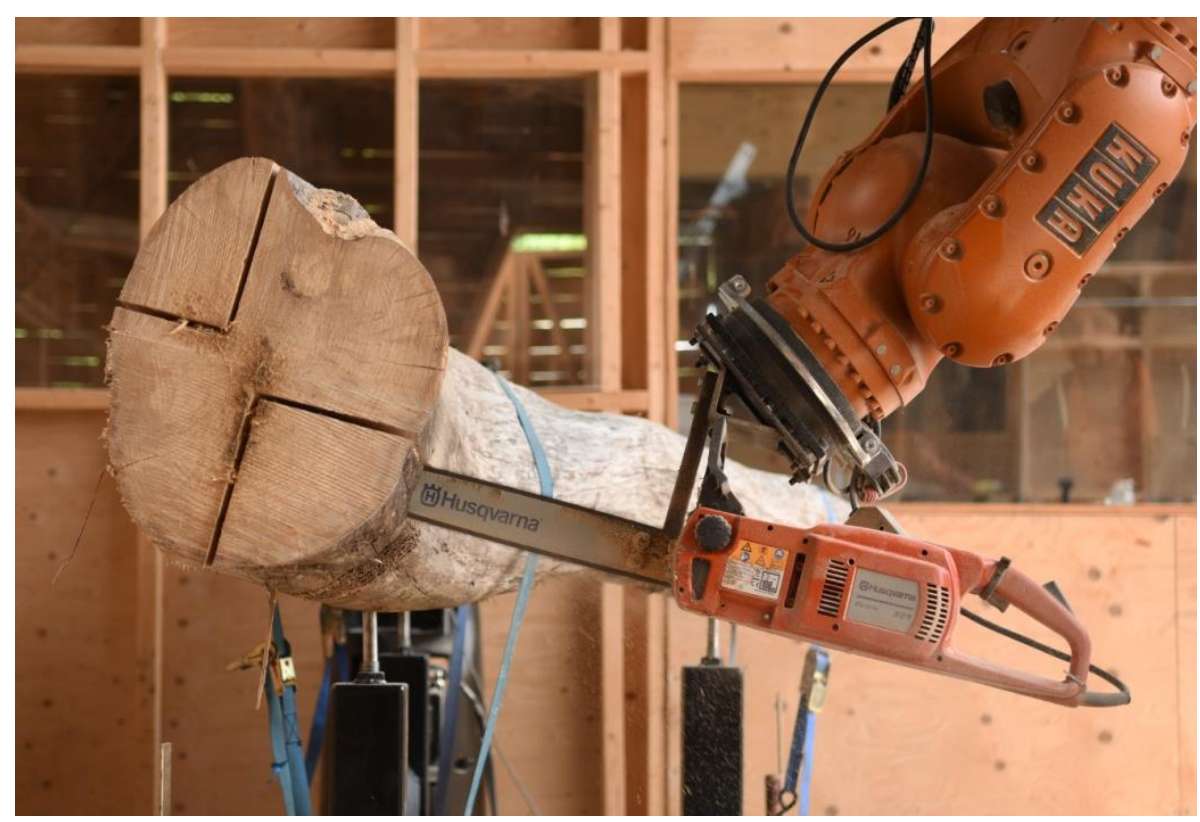

Fig. 4. Weapon of choice - chainsaw end effector used during 'Chainsaw Choreographies'

In the hands of a human operator, the chainsaw becomes a vigilant act of averaging out and balance - the operator's body offering the damping needed to maintain a straight cut through the saw's violent vibrations. Resistance is evaluated throughout, and cut speed adjusted instinctually, with the operator free to move continually in the cut's 
plane. Directed by the robotic arm, these conventional tools gain an augmented level of precision and control. The behaviour of the tool has changed - and the modes in which the tool may be used fundamentally altered. Some of these deviations reveal themselves instantly and are obvious, ${ }^{3}$ others require deeper analysis and are observed through careful examination of film and various surveying mediums.

The chainsaw is installed as an end effector as-found. Two holes through the saw's bar allow it to be fixed while preventing continuous fine tuning of the chain's tension impacting its calibration. The bandsaw's reinvention is taken a step further. Disassembling an off-the-shelf saw, a bespoke bandsaw was crafted from its remains. Welding together a minimal frame, all non-essential components were left off and its cutting gate widened to maximise cutting access. Wielded by the robotic arm, the bandsaw reverses its traditional relationship to the material it works on - traveling towards it.

Rules emerge governing the forms to be produced by each tool. As for the Japanese carpenter whose complex joints are developed from a series of planar cuts owing to the plane of their pullsaw, each cut in 'Chainsaw Choreographies' is governed by the chainsaw's flat bar - its dimension dictating the size of components and joints. ${ }^{4}$ Enacting invisible instructions, the robotic arm has no need to set out its cuts - avoiding a timeconsuming process on complex timber. Where the saw is unable to finish a joint unaided due to reach or depth, it is used to provide markings for hard finishing. Working with a thin flexible blade, the bandsaw is able to carefully make singly curved cuts. Carefully sculpted form and complex joint becoming one.

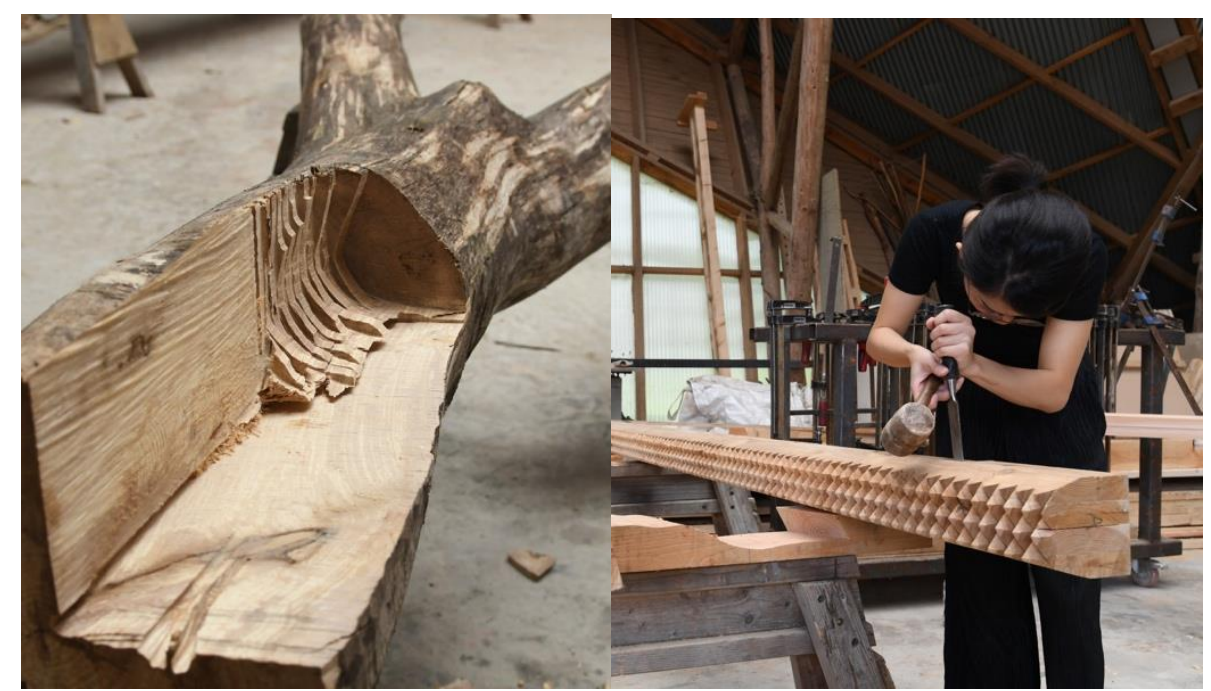

\footnotetext{
${ }^{3}$ While the robotic arm operates with sub millimeter precision, through close observation, the chainsaw was found to resonate up to $4 \mathrm{~mm}$ due to the arm's inflexibility and lack of damping.

${ }^{4}$ The Chainsaw uses a bar of approximately $400 \times 60 \times 4 \mathrm{~mm}$, while the bandsaw's kerf is much thinner and restricted by the $400 \mathrm{~mm}$ cutting gate between its two wheels.
} 
Fig. 5. Analog finishing is applied through both workshops to complete joinery for which the robotic arm has set out the extents (left - kerf to be removed) and to ensure a tight join (right).

\subsection{Robot Language and Logistics}

Restricted by the orientation of existing software packages towards industrial operations, the pace and range of our experimentation demands the development of bespoke toolpath generators. Parameter based offline programming is developed through KUKA|prc's inverse kinematic solver - allowing complex sequences of operations to be programmed by inexperienced participants. For the chainsaw, toolpaths are generated from a minimal input of two curves - the first defining the extreme cutting edge of the saw and the second governing its orientation. For the bandsaw, toolpaths are generated by extracting medial curves from each surface of complex objects modelled in $3 \mathrm{D}$. Retract heights are generated from these guide curves to ensure safe entry and exit from the material, and in areas containing radiuses and curvature, a greater density of planes are generated in order to ensure both smooth and precise cutting.

As the human operator carefully considers their physical orientation to both tool and workpiece, the mounting of each end-effector to the robotic arm and methods for stock holding are thoroughly thought through in each instance - balancing the need for workpiece stability and maximum access. Constraints of the arm itself are considered in parallel - the chainsaw for instance mounted at an angle of 45 degrees to the arm's 6th axis to avoid wrist singularities

While the robotic arm allows each saw to approach the workpiece in ways a human operator may not manage, the saw may still struggle through the cut owing to characteristics of their blades. ${ }^{5}$ This correspondence between analog and digital workmanship becomes an important component of our investigations - identifying the unique potential offered by mounting each tool as an end-effector.

\section{Performative Nature of Manufacturing}

The natural habitat of robots is the controlled environment of the factory. Their natural movement strategy the utilitarian tool-path described by the typical requirement to move in prescribed lines or arcs, and optimised for economic efficiency. Investigating the principles underlying Japanese joining we actively pursue the potential of allowing operations to deviate from the restrained motion paths of industrial applications. Explorations of controlled movement borrowed from the realm of martial arts and performance infect the work with an exciting spatial complexity.

5 Chainsaws for example may be mounted with ripping (for cutting along grain) or crosscutting (for cutting across grain) chains - each possessing a different tooth profile. Impractical to switch between each operation, the arms feed rates are instead adjusted to compensate - simulating the human operator. With the bandsaw, a trade off is made between speed of cutting (requiring a large tooth pitch) and minimum turning radius (requiring a finer band/tooth). 

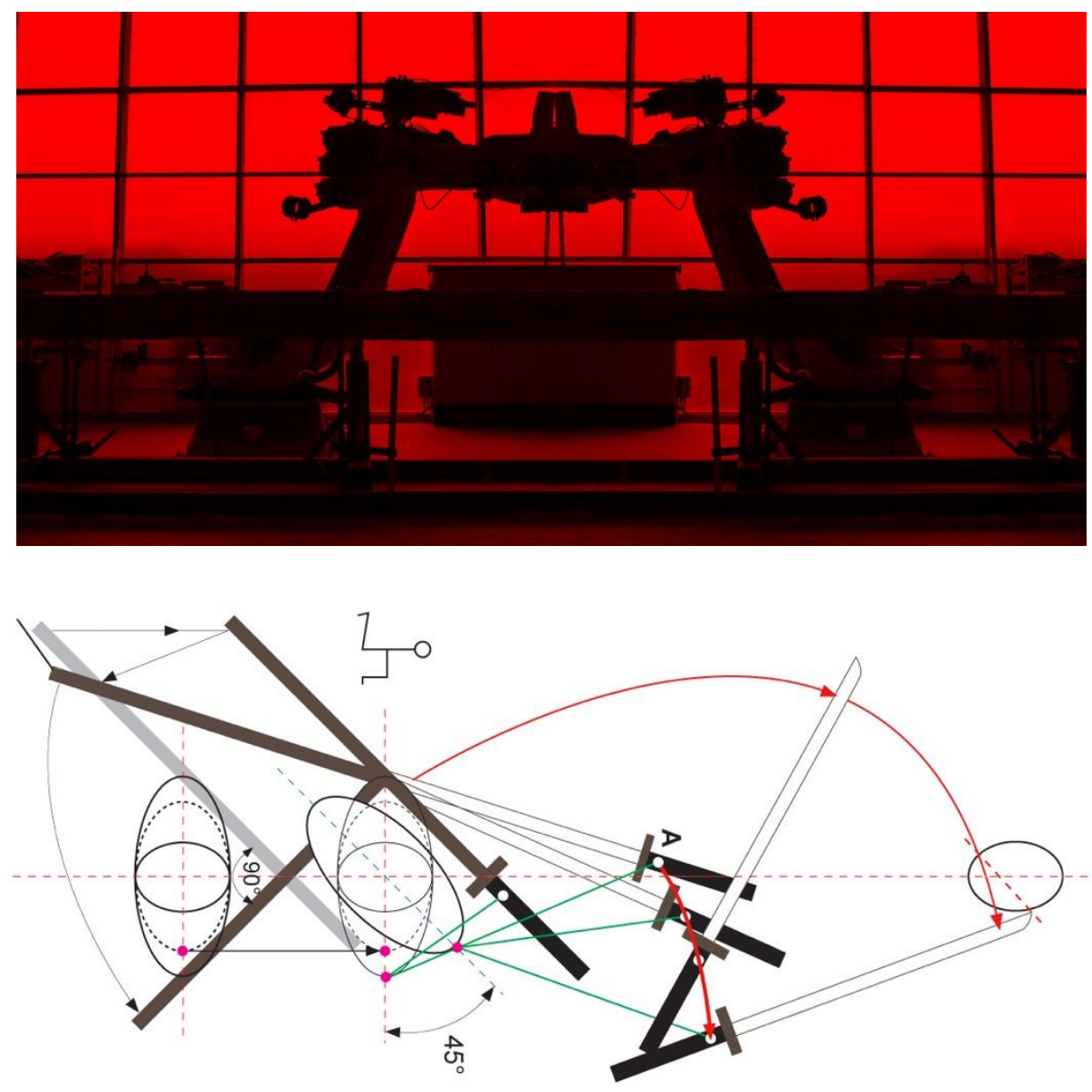

Fig. 6. In Robotic Fabrications, typical lead-in sequences are transformed, taking inspiration from different stances observed in the practice of Iaido (Below [7]).

Operating within the depths of the code, these prescribed movement sequences link code segments that relate to the more functional operations where the chainsaw directly engages with the timber. These highly choreographed movement arrangements serve to connect the primary movement sequences to each other in space and are mainly applied through linking commands.

The conventional home and repose positions became informed by terminology and principle translated from the art of Iaido [8]. This traditional Japanese martial art of the sword strives for smooth, controlled movements of drawing the sword from its scabbard aiming to strike or cut with a dynamic and elegant action. Typical lead-in sequences were transformed by different stances - strategic standing positions from which an attacking or defensive move originates. These were closely observed and respectfully translated towards movement sequences for robot and chainsaw. 
These inserted motions produced an amalgamation of technology, timber connections and martial arts, generating an exciting spatial complexity and developing an artistic engagement within the world of physical production.

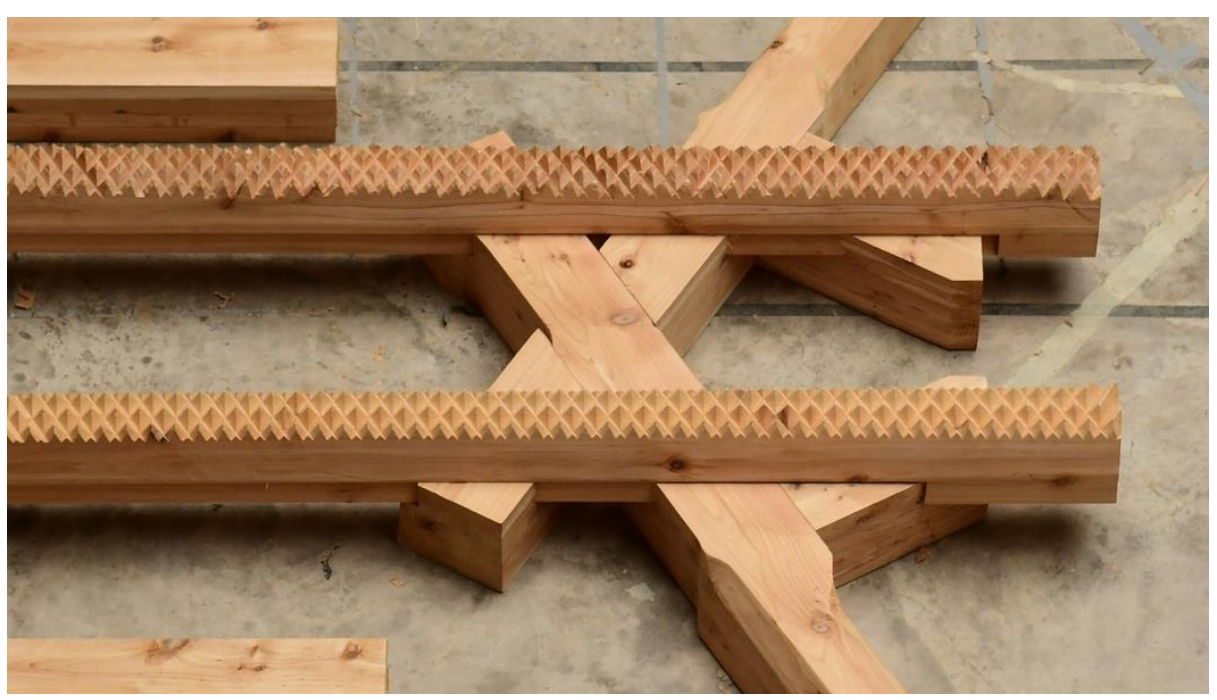

Fig. 7. Ornament and form are explored alongside joining strategies.

\section{Conclusion}

Through this project we are in search of serendipitous moments of fusion between tool, timber construction and performance. These choreographies situate the precision and repeatability inherent in robotics in relation to traditional fabrication techniques and hijacked tools, defining gestural motions not born from efficiency and suggestive but instead of cultural references and occasionally mischief. We are vigorously resisting ideas of full automation - instead we forcefully extend and invent ways to increase the level of interaction with our tools. Sustaining the notion of tools as extensions of our bodily self, the idea that they perform fully independently -as a phantom limb- only advances the disconnect between design and fabrication.

Encouraging a dialog between haptic and scripted operations lets us circumnavigate their individual shortcomings and produces workflows that work complimentary.

Implementing various tools regularly used in conventional timber fabrication, our aim is to use robotic technology not forcefully, for power, repeatability or willful formalism, but delicately, for the strategic augmentation of a naturally complex material. We are concerned with direct applications for industry, but also with the inherent possibilities of beauty and power, theatre and magic [9]. Ultimately, we operate in a territory where robotics in architecture, art, and design can construct a highly original reality and be seen as a powerful instrument for creative speculation. 


\section{$5 \quad$ References}

1. Sato, H., Nakahara, Y.: Trans: Koichi Paul Nii: The Complete Japanese Joinery. Hartley \& Marks, Vancouver (1995)

2. Mollica, Z., Self, M.: Geometric Strategies for Exploiting Inherent Material Form. In: Advances in Architectural Geometry. vdf Hochschulverlag AG an der ETH, Zurich (2016).

3. kkaarrlls homepage, http://www.kkaarrlls.com/index.php?feature=editions,7Xstool, last accessed 18/05/12.

4. Pawlofsky, T.: Rhino2krl. In: Rob|Arch 2012, Brell-Çokcan S., Braumann J. (eds). Springer, Vienna (2013).

5. Yuan, P., Chair, H.: Robotic Wood Tectonics. In: Fabricate 2017, by Menges, A., Sheil, B., Ruairi, G., Marilena, S.. UCL Press, London (2017).

6. Luke Johns, R., Foley, N.: Bandsawn Bands: Feature-Based Design and Fabrication of Nested Freeform Surfaces in Wood. In: Robotic Fabrication in Architecture Art and Design. Springer, Switzerland (2014).

7. "Nuki Tsuke" [digital image] (n.d.) Retrieved from https://shinbukan-paris-iaijo.tumblr.com, last accessed 18/05/08.

8. Salmon, G.: Kendo: A Comprehensive Guide to Japanese Swordsmanship, Tuttle Publishing (2013).

9. Davies, K. Vercruysse, E., Svilans, T., Dodd, I.: Instruction and Instinct, Performing within Unordered Complexity. In: Robotic Fabrication in Architecture, Art and Design. Springer, Switzerland (2014). 